infection) and HCV antibodies in whole blood. Enzyme immunoassays were used for detecting HBV core (anti$\mathrm{HBc}$ ) and surface (anti-HBs) antibodies (lifetime infection: $\mathrm{HBsAg}$ or anti-HBc positivity without anti-HBs). Risk factors analyses controlled for potential confounders using log-binomial regression.

Results The prevalence of active and lifetime HBV were $8.8 \%$ and $37.7 \%$, respectively. Only two men were HCV-positive $(0.98 \%)$. Both current $(16.7 \%$ versus $6.4 \%, \mathrm{p}<0.0001)$ and lifetime $(66.7 \%$ versus $28.8 \%, p<0.0001)$ HBV infection were more prevalent among MSM aged $\geq 30$ years compared to younger subjects. Sexual intercourse under the effect of drug or alcohol, and living in couple were also associated with both current and lifetime HBV.

Conclusion HBV is frequent in this population, especially in older MSM who are more likely to have started their sexual life before the initiation of MSM-specific interventions in Benin (2008). HCV prevalence was low, likely because of the absence of injectable drug use and the fact that all participants were HIV-negative. HBV vaccination, offered free of charge to children in Benin since 2005, should also be systematically offered to MSM susceptible to this infection.

\section{P138 EVALUATING OPT-OUT STI TESTING AT ADMISSION WITHIN A SHORT-TERM CORRECTIONAL FACILITY LOCATED IN ALBERTA, CANADA}

${ }^{1}$ A Reekie*, ${ }^{2} \mathrm{~J}$ Gratrix, ${ }^{3} \mathrm{R}$ Ahmed, ${ }^{2} \mathrm{P}$ Smyczek. 'School of Public Health, University of Saskatchewan, Saskatoon, Canada; ${ }^{2}$ STI Services, Alberta Health Services, Edmonton, Canada; ${ }^{3}$ Department of Medicine, University of Alberta, Edmonton, Canada

10.1136/sextrans-2021-sti.249

Background Incarcerated Canadians entering into correctional facilities experience higher rates of sexually transmitted infections (STI) than the general population. Yet, testing across correctional facilities remains inconsistent and symptom- or risk-based. Thus, STI prevalence is likely underestimated, and the number of undiagnosed individuals remains high. In response, Alberta implemented universal opt-out STI (chlamydia, gonorrhea, syphilis)/HIV screening at admission in a short-term correctional facility for all individuals $\leq$ 35 years.

This study evaluates opt-out screening at admission to provide Canadian-specific data as a comparative to current opt-in (symptom/risk-based) testing strategies. Specific outcomes were determined: (1) the uptake of opt-out screening, (2) reasons for opt-out screening non-completion, (3) STI/HIV positivity rates and (4) treatment completion rates.

Methods A cross-sectional, retrospective analysis of opt-out screening outcomes between March 2018 and February 2020 was completed. Test data was extracted from admission linelists, laboratory data and communicable disease treatment data. Descriptive statistics were used to stratify test data by STI, gender, age group, and date for univariate analysis.

Results Opt-out screening was offered to the majority (96.7\%) of admissions, while opt-out screening non-completion was largely attributed to patient decline (67.4\%). Despite low testing uptake (31.2\%), opt-out screening achieved high positivity rates $(14.9 \%$ chlamydia, $10.8 \%$ gonorrhea, $29.5 \%$ syphilis and $0.3 \%$ HIV) and treatment completion rates $(94.1 \%$ overall). Furthermore, $52.6 \%$ of opt-out cases were asymptomatic.

Conclusions Although limited by retrospective analysis, opt-out screening at admission is a feasible strategy to increase STI/ HIV testing and detect asymptomatic cases in a correctional setting. Communicative strategies within the facility will be critical to increase testing uptake among patients. Intensified screening and subsequent treatment can impact community transmission while reducing healthcare accessibility barriers for a vulnerable population.

Further, implementing opt-out STI/HIV screening in other high-risk settings, such as mental health and addictions, can be an effective case-finding strategy for outbreak management.

\section{P139 A PHYLOGENOMIC SURVEY OF DISSEMINATED GONOCOCCAL INFECTION ISOLATES IN THE UNITED STATES (2019-2020)}

'B Raphael ${ }^{*}$, 'J Cartee, 'S Joseph, 'S Sharpe, 'B Roland, 'K Gernert, 'M Schmerer, ${ }^{1} \mathrm{~A}$ Ridpath, ${ }^{1} \mathrm{~L}$ Quilter, ${ }^{1} \mathrm{~S} S \mathrm{St}$ Cyr, ${ }^{1} \mathrm{~K}$ Schlanger, ${ }^{2} \mathrm{E}$ Burgess, ${ }^{2} \mathrm{~S}$ Dietrich, ${ }^{1} \mathrm{~K}$ Bernstein, ${ }^{1} \mathrm{E}$ Kersh. ${ }^{1}$ Centers For Disease Control and Prevention, Atlanta, USA; ${ }^{2}$ Michigan Department of Health and Human Services, Lansing, USA

\subsection{6/sextrans-2021-sti.250}

Background In December 2019, Centers for Disease Control and Prevention (CDC) released a Dear Colleagues Letter describing best practices for investigation of Disseminated Gonococcal Infection (DGI) cases which stressed the importance of culture from sterile and mucosal sites for additional analysis including genomic sequencing. The objective of sequencing was to understand the diversity of DGI isolates and explore the extent of genetically related isolate clusters in the US.

Methods Between 2019 and 2020, CDC conducted sequencing on submitted DGI isolates using the Illumina MiSeq platform. Separately, the Michigan Department of Health and Human Services sequenced isolates submitted by laboratories in Michigan. Core genome Single Nucleotide Polymorphism (SNP) analysis was performed using snippy and the FA19 strain as a reference sequence. Detection of selected antibiotic resistance markers and multilocus sequencing typing (MLST) alleles was conducted using the Gonorrhea AMR Profiler and Typing Tool.

Results We analyzed 45 isolates submitted by 7 states representing a total of 12 MLST sequence types (ST). The majority $(66.7 \%)$ of these STs are among the top 25 STs found in a representative dataset of isolates sequenced from the Gonococcal Isolate Surveillance Program in 2018. We also identified 3 clusters of genetically related DGI isolates including 14 isolates from Michigan with a mean difference of 22 SNPs (range: 432), 5 isolates from North Carolina with a mean difference of 12 SNPs (range:8-28), and 2 isolates from North Carolina differing by 11 SNPs. All genomes examined contained wild type 23S rRNA markers (positions 2059 and 2611) and nonmosaic penA alleles. Notably, $>85 \%$ of the sequences contained the porB1a allele, previously associated with serum resistance.

Conclusion Isolates recovered from DGI cases are genetically diverse and frequently share STs associated with circulating isolates recovered from uncomplicated gonorrhea. The detection of DGI isolate clusters highlights the need for further epidemiological investigation. 